This is the peer reviewed version of the following article:: Angew. Chem. Int. Ed., 2018, 57, 2464-2468, which has been published in final form at https://onlinelibrary. wiley.com/doi/full/10.1002/anie.201712088. This article may be used for non-commercial purposes in accordance with Wiley Terms and Conditions for Use of Self-Archived Versions.

\title{
The Catalytic Asymmetric Mukaiyama-Michael Reaction of Silyl Ketene Acetals with $\alpha, \beta-$-Unsaturated Methyl Esters
}

\author{
Tim Gatzenmeier, Philip S. J. Kaib, Julia B. Lingnau, Richard Goddard and Benjamin List*
}

\begin{abstract}
Unsaturated esters are readily available but challenging substrates to activate in asymmetric catalysis. We now describe an efficient, general, and highly enantioselective Mukaiyama-Michael reaction of silyl ketene acetals with $\alpha, \beta$-unsaturated methyl esters, catalyzed by a silylium imidodiphosphorimidate (IDPi) Lewis acid.
\end{abstract}

Michael additions of enolate equivalents to $\alpha, \beta$-unsaturated carbonyl compounds are widely applied carbon-carbon bond forming reactions and the development of catalytic asymmetric methods has been the subject of intensive research over the past decades. ${ }^{[1]}$ While $\alpha, \beta$-unsaturated aldehydes and ketones readily engage in various catalytic enantioselective Michael additions via iminium ion, Brønsted acid, or Lewis acid catalysis, ${ }^{\left[{ }^{[} \bar{j},[\bar{j}],[\bar{i}]\right.} \alpha, \beta$-unsaturated esters the original substrates in Michael's seminal study in $1887^{[5]}$ have proven to be particularly challenging substrates for such reactions. Very recently, Mayr and coworkers provided an explanation for their low reactivity by systematically quantifying the electrophilicity of a wide range of common Michael acceptors: ${ }^{[\overline{6}]}$ Indeed, $\alpha, \beta$-unsaturated esters in general, and cinnamates in particular, rank among the very least electrophilic substrates (Figure 1A). However, because $\alpha, \beta$-unsaturated esters are naturally abundant, industrially relevant, and inexpensive or readily available, they are highly attractive substrates for Michael additions. Here we show that a silylium imidodiphosphorimidate (IDPi) Lewis

[*] T. Gatzenmeier, Dr. P. S. J. Kaib, J. B. Lingnau, Dr. R. Goddard, Prof. Dr. B. List

Max-Planck-Institut für Kohlenforschung

Kaiser-Wilhelm-Platz 1, 45470 Mülheim an der Ruhr (Germany)

E-mail: list@kofo.mpg.de

[**] Funding from the Max-Planck-Society, the European Research Council (Advanced Grant CHAOS) and the DFG (Leibniz Award to $\mathrm{BL}$ ) is gratefully acknowledged. This work is part of the Cluster of Excellence RESOLV (EXC 1069) funded by the DFG. We thank Sunggi Lee and Francesca Mandrelli for helpful discussions and sharing of reagents. We also thank the technicians of our group and the analytical departments of our institute for their excellent service. acid catalyzes a highly enantioselective catalytic Mukaiyama-Michael addition of silyl ketene acetals to a range of simple $\alpha, \beta$-unsaturated methyl esters.

In contrast to the desired Michael additions of enolate equivalents, elegant and useful catalytic asymmetric conjugate additions to $\alpha, \beta$-unsaturated esters have previously been developed. Examples include Feringa's copper-phosphoramidite catalyzed conjugate additions of Grignard reagents, ${ }^{[7]}$ Hayashi's rhodium-catalyzed 1,4-additions of boronic acids, ${ }^{[8]}$ and Pfaltz' cobalt-catalyzed conjugate reductions. ${ }^{[9]}$ Organocatalytic, enantioselective Stetter reactions with $\alpha, \beta$-unsaturated esters have also been described. ${ }^{[10]}$ Furthermore, to circumvent the poor reactivity of $\alpha, \beta$-unsaturated esters, more electrophilic $\alpha, \beta$ unsaturated $\mathrm{N}$-acyl oxazolidinones, $\mathrm{N}$-acyl imides, $\mathrm{N}$-acyl imidazolides, thioamides, a-ketophosphonates, alkylidene malonates, or perfluorinated esters ${ }^{[11]}$ have been suggested as ester surrogates in Michael-type additions, but such reagents are inherently less atom- and step-economic. To activate $\alpha, \beta$ unsaturated esters themselves, only few Lewis acidic catalysts have been reported, including Corey's oxazaborolidine derivatives and bifunctional hydrogen bonding catalysts. ${ }^{[12],[13]}$

A. The Challenge: Catalytic Asymmetric Michael Addition of Enolates to Weakly Electrophilic $\alpha, \beta$-Unsaturated Esters

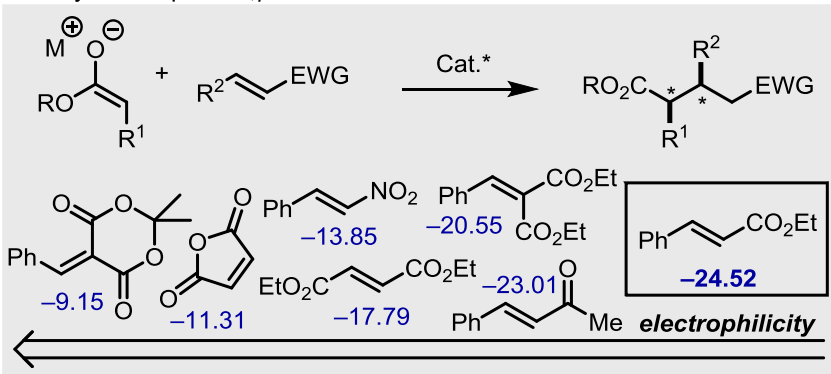

B. This Work: Asymmetric Mukaiyama-Michael Reaction of Silyl Ketene Acetals to $\alpha, \beta$-Unsaturated Methyl Esters

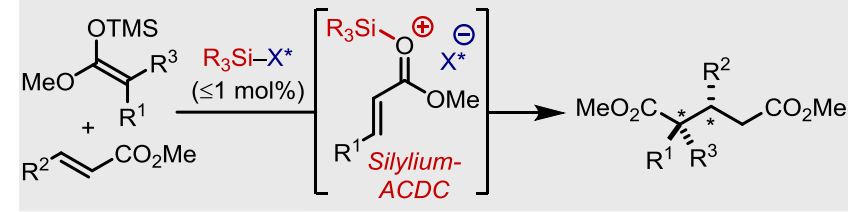

Figure 1. Asymmetric catalysis of the Michael reaction. 
Within our research program of exploring the potential of asymmetric counteranion-directed silylium Lewis acid catalysis (silylium-ACDC), ${ }^{[14],[15]}$ we recently focused our attention on the activation of $\alpha, \beta$-unsaturated esters in catalytic asymmetric Diels-Alder reactions with cyclopentadiene. ${ }^{[16]}$ While these studies suggested sufficient reactivity of our silylium Lewis acid catalysts, high enantioselectivities were only obtained with 9fluorenylmethyl esters. These substrates are electronically nonactivated, but prone to strong dispersion interactions. ${ }^{[17]}$ Striving to make the simplest and most readily available methyl esters accessible as substrates, we became highly interested in exploring their utility in silylium-ACDC and specifically in the asymmetric Mukaiyama-Michael reaction (Figure 1B).

We began our study with the asymmetric conjugate addition reaction of methyl trans-cinnamate (1a) with commercial silyl ketene acetal (SKA) 2a (Table 1). ${ }^{[18]}$ In preliminary studies of this model reaction, we found that Lewis acids derived from our chiral disulfonimides (DSI), ${ }^{[15]}$ or binaphthyl-allyl-tetrasulfone (BALT) $\mathrm{C}-\mathrm{H}$ acids, ${ }^{[16]}$ were either insufficiently reactive as catalysts or gave very low enantioselectivities (see SI for further detail). In contrast, encouraging results were obtained with our recently developed IDPi acids ${ }^{[19]}$ 4a-e (Table 1, entries $\left.1-5\right)$. This catalyst motif combines very high acidity with a confined threedimensional structure, and generates a $\mathrm{C}_{2}$-symmetric anion upon deprotonation.

Table 1. Reaction development. ${ }^{[a}$

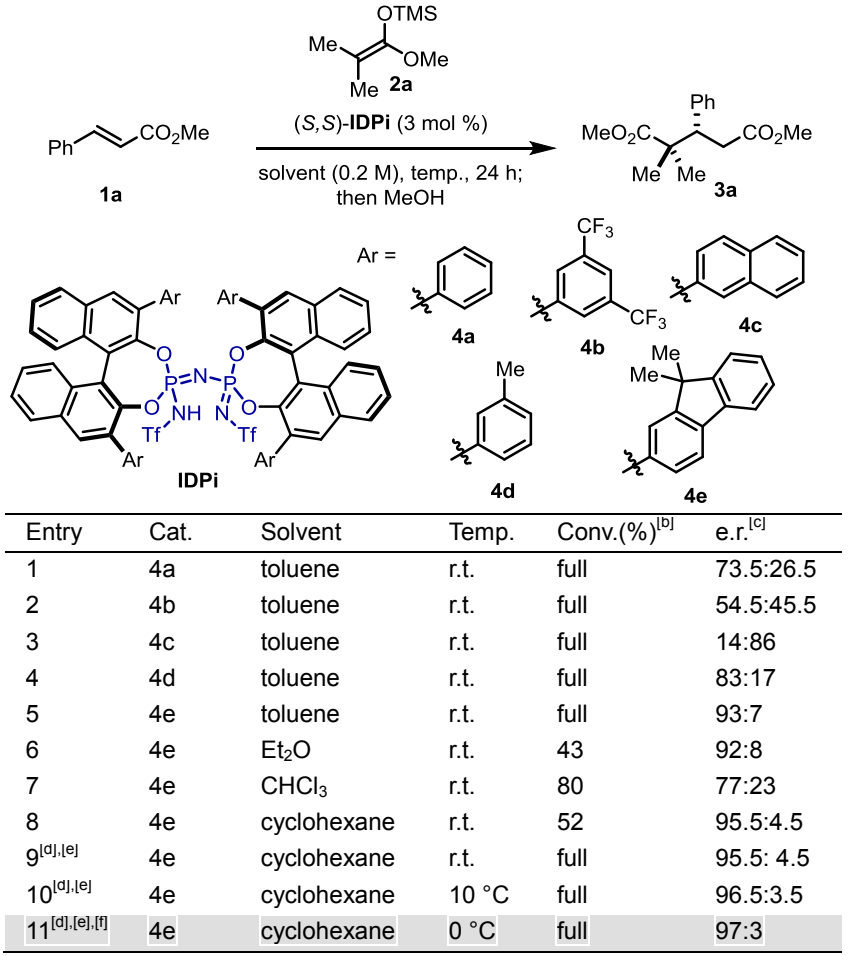

[a] Reactions were performed on a $0.02 \mathrm{mmol}$ scale. [b] Determined by ${ }^{7} \mathrm{H}$ NMR. [c] Determined by HPLC. [d] Freshly prepared and purified SKA 2a was used. [e] $1 \mathrm{~mol} \%$ of catalyst. [f] $12 \mathrm{~h}$ reaction time.

Of the investigated IDPi acids, $4 \mathbf{e}$ was identified as the best catalyst (entry 5). Further optimization resulted in very high enantioselectivity but only moderate conversion (entry 8). A remarkable enhancement in reactivity was observed, when instead of commercially available SKA 2a, a freshly synthesized and purified reagent was used. ${ }^{[20]}$ This allowed us to obtain full conversion with only $1 \mathrm{~mol} \%$ of catalyst at $0{ }^{\circ} \mathrm{C}$ after $12 \mathrm{~h}$ (entry 11 ), compared to the $52 \%$ conversion at r.t. with $3 \mathrm{~mol} \%$ of catalyst and $24 \mathrm{~h}$ of reaction time obtained with the commercial reagent (entry 8 ). We attribute this effect to trace impurities of diisopropylamine in the commercially available SKA that potentially deactivate the catalyst.

Table 2. Ester scope. ${ }^{[a]}$

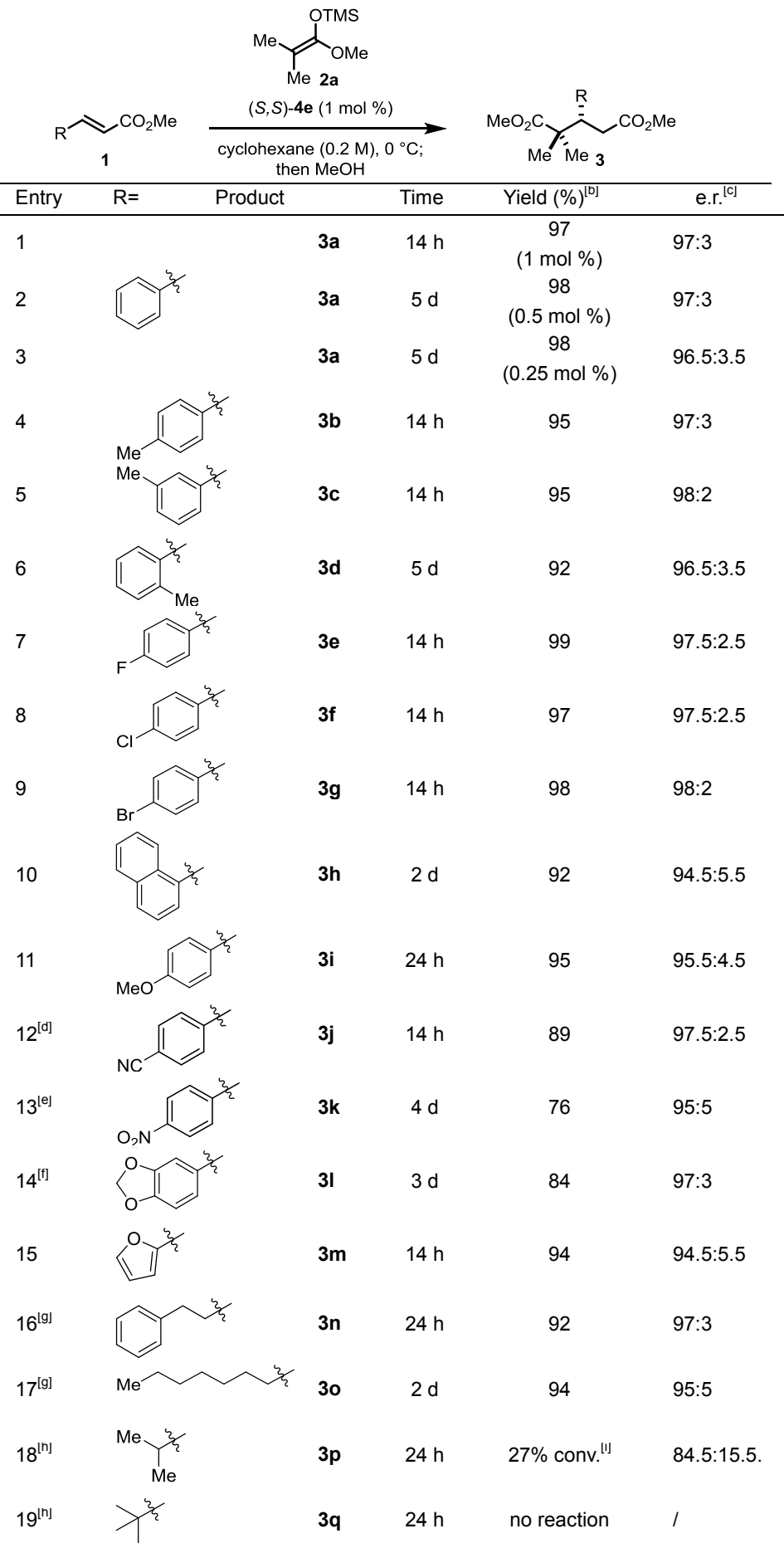

[a] Reactions were performed on a $0.2 \mathrm{mmol}$ scale with 3.0 equivalents of SKA 2a for the specified period of time. [b] Isolated yield. [c] Determined by HPLC. [d] In p-xylene. [e] In toluene/1,4-dioxane (3:1) at r.t.. [f] In $m$-xylene. [g] In methylcyclohexane at $-40{ }^{\circ} \mathrm{C}$. [h] with $5 \mathrm{~mol} \%$ catalyst and at $40{ }^{\circ} \mathrm{C}$. [i] Determined by ${ }^{1} \mathrm{H}$ NMR. 
With optimal conditions in hand, we next investigated the scope of the reaction by employing a variety of 3-aryl and 3alkyl methyl trans-acrylates (Table 2). With model substrate $1 \mathrm{a}$, the catalyst loading could even be lowered to $0.25 \mathrm{~mol} \%$ (entries 1-3) giving consistently excellent yields of product 3a with prolonged reaction time. Differently substituted arenes (3b-d, 3h) as well as halogenated substrates (3e-g) gave the desired products in excellent yields and enantioselectivities. Further, both electron-rich $(\mathbf{3} \mathbf{i}, \mathbf{3} \mathbf{I})$ and electron-deficient substrates $(\mathbf{3 j}, \mathbf{3 k})$ were well tolerated with slightly modified conditions. Also heterocyclic product $3 \mathrm{~m}$ could be obtained with good results. As linear 3-alkyl trans-acrylates are

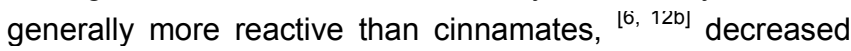
temperatures were necessary to obtained the desired products with high enantiocontrol (3n, 30). Y-Branched substrates (entry 18-19) were found very unreactive and only very low or no conversion was detected even with increased catalyst loadings and temperatures.

We also studied other cinnamates, including the corresponding ethyl- and benzyl esters, free cinnamic acid, cinnamoyl chloride and methyl cis-cinnamate. Interestingly, ethyl trans-cinnamate gave strongly diminished enantioselectivity (e.r. $77.5: 22.5)$ and reactivity (89\% yield, 3 d reaction time). When methyl cis-cinnamate (ZIE >99:1) was used, only very low conversion ( $55 \%$ yield) was detected after 5 days and the opposite enantiomer was enriched (e.r. 62.5:37.5). All other tested substrates gave no conversion. These results are consistent with a scenario in which catalyst $4 \mathrm{e}$ exhibits an ideally shaped chiral pocket to accommodate the geometry of trimethylsilylated $\alpha, \beta-$ unsaturated trans-methyl esters. While modifications of the 3-position are well tolerated, distortion of this geometry would result in either decelerated or complete shutdown of catalysis.

The scope of silyl ketene acetals was explored next (Table 3). With cyclic SKA nucleophiles (2b-d), the corresponding products were obtained with high yields and enantioselectivities. With a-mono-substituted silyl ketene acetals, the reaction outcome was found to significantly depend on the type of SKA employed. Both isomeric SKAs (E)-2e (E/Z 4:1) and (Z)-2e (E/Z 1:19) gave excellent enantioselectivities (e.r. $\geq 98.5: 1.5$ ), while favoring opposite diastereomers: $(E)-\mathbf{2 e}$ afforded syn-product 5d (d.r. 12:1), while $(Z)-2 e$ predominantly gave the corresponding antiproduct $5 \mathrm{e}$ (d.r. 1.6:1). Intriguingly, the reaction failed to provide any increased diastereoselectivity with all SKA variants when triflimide $\left(\mathrm{HNTf}_{2}\right)$ was used as an achiral catalyst, highlighting the additional benefit from a confined reaction environment beyond enantiocontrol (see the SI for further detail). Isopropyl-substituted SKA $(E)-\mathbf{2 g}$ was found to provide product $\mathbf{5 f}$ with excellent diastereoselectivity and enantioselectivity (entry 7 ). The observed preference for the syn- or anti-diastereomer in relation to the $(E)$ - or $(Z)$-enolate geometry is in agreement with that observed by Evans et al. in their Mukaiyama-Michael reactions of $\alpha, \beta$-unsaturated $N$ acyl oxazolidinones. ${ }^{[21]}$

Table 3. Silyl ketene acetal (SKA) scope. ${ }^{[a]}$

(d.r.) $)^{[\mathrm{c}]}$

[a] Reactions were performed on a $0.2 \mathrm{mmol}$ scale with 3.0 equivalents of SKAs. [b] Isolated yield. [c] e.r. and d.r. by HPLC. [d] 2 mol \% catalyst. [e] $5 \mathrm{~mol} \%$ catalyst.

Toward understanding the reaction mechanism, we became interested in the nature of the initial reaction product before methanolysis. Besides the previously reported [2+2] or [4+2] intermediates ${ }^{[\overline{Z Z j},[\bar{z} i a]}$ with ketene acetals, we also anticipated an open-chain intermediate as proposed in other silylium

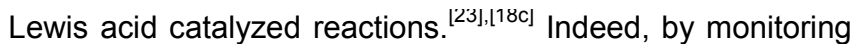
the reaction by NMR spectroscopy, we could identify and characterize silyl ketene acetal D (Figure 2) as the initial reaction product. Interestingly, a Z/E-ratio of $>99: 1$ of product D was observed, consistent with an s-cis conformation of the reacting $\alpha, \beta$-unsaturated ester in the carbon-carbon bond forming transition state. Accordingly, a tentative catalytic cycle is suggested, which is initiated by the protonation of the SKA with the IDPi precatalyst, furnishing silylated ester A. Silicon-transfer to the substrate then leads to the activated chiral ion pair $\mathbf{B}$, which reacts with the nucleophile to provide a doubly-silylated intermediate $\mathbf{C}$. Silylation of the next substrate molecule could then occur either directly or via other esters of the generic type A. Since intermediate D is a potential nucleophile itself, we tested whether reduced amounts of the starting SKA 2a would cause oligomerizations by subsequent Michael addition of intermediate $\mathbf{D}$ to unsaturated ester 1a. However, we found that even only 1.1 equivalents of SKA $\mathbf{2 a}$ gave a clean reaction profile and the same yield after prolonged reaction time. Also sub-stoichiometric amounts of $\mathbf{2 a}$ did not result in 
any significant side product formation even after prolonged reaction times at room temperature. Increased steric hindrance at silyl ketene acetal $\mathbf{D}$ and as a consequence lower reactivity may account for this observation.

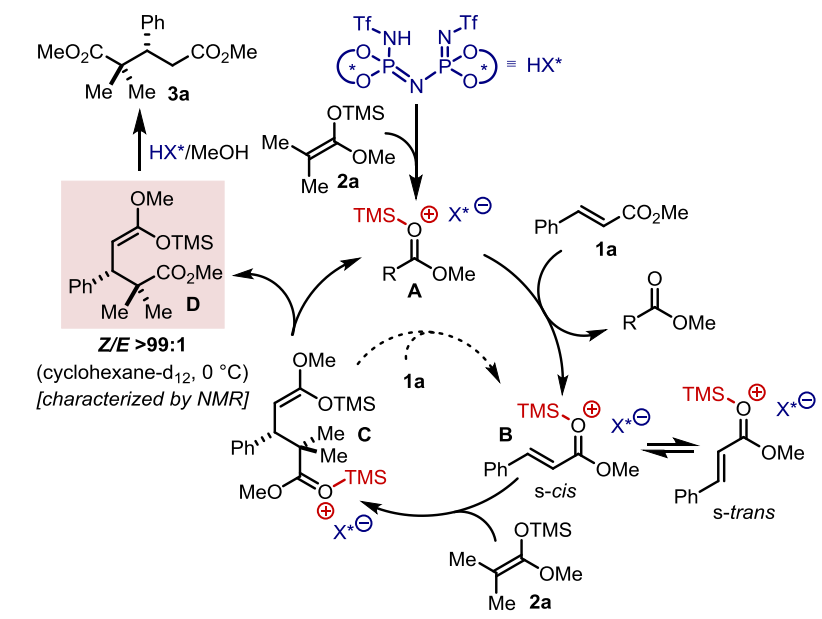

Figure 2. Proposed reaction mechanism.

Generally, the isolated yields correlated well with the employed amounts of SKA (see SI for further details), and the self-healing features of silylium Lewis acid catalysis $^{[15 b], 116]}$ also allowed us to conduct the reaction in non-dried solvent and without requirement of an inert gas atmosphere. Product $\mathbf{3 a}$ was isolated in identical yield and enantioselectivity under such conditions.

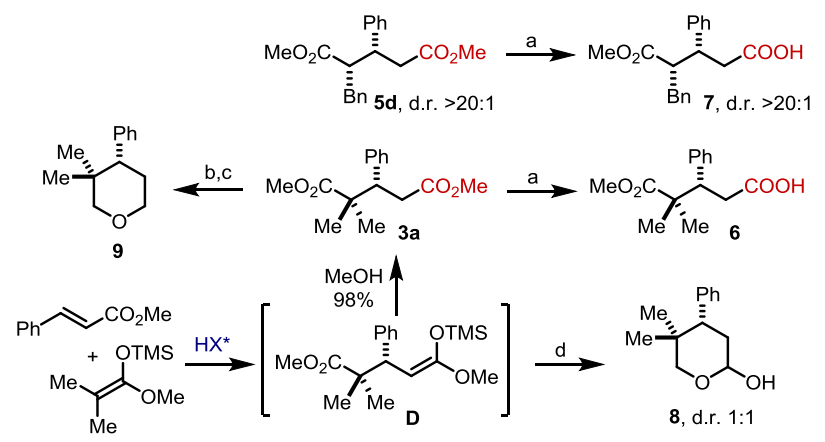

Figure 3. Selective product derivatizations. a) aq. $\mathrm{NaOH}$ (5 equiv.), $\mathrm{MeOH} / \mathrm{THF}(2: 1), 0^{\circ} \mathrm{C}$ to r.t., $1 \mathrm{~h}$, quant.; b) $\mathrm{LAH}\left(1.5\right.$ equiv.), THF, $0{ }^{\circ} \mathrm{C}$ to r.t., $3 \mathrm{~h}$, quant.; c) $\mathrm{TsOH}(20 \mathrm{~mol} \%), \mathrm{MgSO}_{4}$, toluene, $120^{\circ} \mathrm{C}, 16 \mathrm{~h}, 97 \%$; d) $\mathrm{LAH}$ (1.5 equiv.), $0{ }^{\circ} \mathrm{C}, 3 \mathrm{~h}, 75 \%$.

As differentiation of the two product ester groups would be highly attractive for further transformations, we investigated possibilities for selective derivatizations (Figure 3). Gratifyingly, we found that simple saponification with aq. $\mathrm{NaOH}$ provides a very high degree of selectivity for the less sterically hindered ester group. Thus, both products $\mathbf{3 a}$ and 5d were converted smoothly into the corresponding monocarboxylic acids 6 and 7 in quantitative yield and in case of product $\mathbf{5 d}$ without epimerization. An alternative and complimentary approach for differentiating the two ester groups of our products would involve the direct utilization of silyl ketene acetal intermediate D. Indeed, we found that adding $\mathrm{LAH}$ instead of methanol to the reaction mixture selectively gave lactol $\mathbf{8}$. Reduction of product $3 \mathbf{3}$ with LAH gave the corresponding diol, which cyclizes under acidic conditions to furnish tetrahydropyran 9 .

In summary, we have developed the first example of an asymmetric Mukaiyama-Michael reaction of $\alpha, \beta$-unsaturated methyl esters. This reaction is enabled by the use of chiral silylium ion-based Lewis acids and delivers high enantioand diastereoselectivity with a broad scope of different substrates. Future work will focus on further applications of this catalytic system and its reaction intermediates for asymmetric synthesis.

Keywords: Mukaiyama-Michael reaction $\cdot$ cinnamates $\cdot$ silyl ketene acetals $\bullet$ organocatalysis $\bullet$ confined Brønsted acid

a) E. M. Carreira, L. Kvaerno, Classics in Stereoselective Synthesis, Wiley, 2009; b) Y. Zhang, W. Wang, Catal. Sci. Technol. 2012, 2, 42-53; c) C. Hui, F. Pu, J. Xu, Chem. Eur. J. 2016, 23, 4023-4036

[2] a) S. P. Brown, N. C. Goodwin, D. W. C. MacMillan, J. Am. Chem. Soc. 2003, 125, 1192-1194; b) Y. K. Chen, M. Yoshida, D. W. C. MacMillan, J. Am. Chem. Soc. 2006 128, 9328-9329; c) S. Bertelsen, P. Diner, R. L. Johansen, K. A. Jørgensen, J. Am. Chem. Soc. 2007, 129, 15361537; d) A. Erkkilä, I. Majander, P. M. Pihko, Chem. Rev. 2007, 107, 5416-5470; e) S. G. Ouellet, A. M. Walji, D. W. C. Macmillan, Acc. Chem. Res. 2007, 40, 1327-1339; f) C. Appayee, S. E. Brenner-Moyer, Org. Lett. 2010, 12, 33563359.

[3] a) D. Nakashima, H. Yamamoto, J. Am. Chem. Soc. 2006 128, 9626-9627; b) T. Sakamoto, J. Itoh, K. Mori, T. Akiyama, Org. Biomol. Chem. 2010, 8, 5448-5454; c) B.M. Yang, P.-J. Cai, Y.-Q. Tu, Z.-X. Yu, Z.-M. Chen, S.-H. Wang, S.-H. Wang, F.-M. Zhang, J. Am. Chem. Soc. 2015 137, 8344-8347; d) N. Momiyama, K. Funayama, H. Noda, M. Yamanaka, N. Akasaka, S. Ishida, T. Iwamoto, M. Terada, ACS Catal. 2016, 6, 949-956.

[4] a) K. Hermann, H. Wynberg, J. Org. Chem. 1979, 44 2238-2244; b) M. Sawamura, H. Hamashima, Y. Ito, J Am. Chem. Soc. 1992, 114, 8295-8296; c) M. Sawamura, H. Hamashima, Y. Ito, Tetrahedron 1994, 50, 4439-4454; d) T. Arai, H. Sasai, K.-i. Aoe, K. Okamura, T. Date, M. Shibasaki, Angew. Chem. Int. Ed. 1996, 35, 104-106; e) Y Hamashima, D. Hotta, M. Sodeoka, J. Am. Chem. Soc. 2002, 124, 11240-11241.

[5] a) A. Michael, J. Prakt. Chem. 1887, 35, 349-356; b) T. Tokoroyama, Eur. J. Org. Chem. 2010, 2010, 2009-2016.

[6] D. S. Allgäuer, H. Jangra, H. Asahara, Z. Li, Q. Chen, H. Zipse, A. R. Ofial, H. Mayr, J. Am. Chem. Soc. 2017, 139, 13318-13329.

[7] a) S. R. Harutyunyan, F. Lopez, W. R. Browne, A. Correa, D. Peña, R. Badorrey, A. Meetsma, A. J. Minnaard, B. L. Feringa, J. Am. Chem. Soc. 2006, 128, 9103-9118; b) S.Y. Wang, S.-J. Ji, T.-P. Loh, J. Am. Chem. Soc. 2007, 129, 276-277; c) S. R. Harutyunyan, T. den Hartog, K. Geurts, A. J. Minnaard, B. L. Feringa, Chem. Rev. 2008, 108, 2824-2852

[8] a) Y. Takaya, T. Senda, H. Kurushima, M. Ogasawara, T. Hayashi, Tetrahedron: Asymmetry 1999, 10, 4047-4056 b) S. Sakuma, M. Sakai, R. Itooka, N. Miyaura, J. Org. Chem. 2000, 65, 5951-5955; c) T. Hayashi, K. Yamasaki, Chem. Rev. 2003, 103, 2829-2844.

[9] a) A. Pfaltz, Acc. Chem. Res. 1993, 26, 339-345; b) U. Leutenegger, A. Madin, A. Pfaltz, Angew. Chem. Int. Ed. $1989,28,60-61$. 
[10] a) M. S. Kerr, T. Rovis, J. Am. Chem. Soc. 2004, 126, 8876-8877; b) J. Read de Alaniz, T. Rovis, J. Am. Chem. Soc. 2005, 127, 6284-6289; c) J. R. de Alaniz, T. Rovis, Synlett 2009, 2009, 1189-1207; d) N. E. Wurz, C. G. Daniliuc, F. Glorius, Chem. Eur. J. 2012, 18, 16297 16301.

[11] a) G. Desimoni, G. Faita, P. Quadrelli, Chem. Rev. 2015 115, 9922-9980; b) N. Kumagai, M. Shibasaki, Chem. Eur. J. 2016, 22, 15192-15200; c) D. Monge, H. Jiang, Y. Alvarez-Casao, Chem. Eur. J. 2015, 21, 4494-4504.

[12] a) E. Canales, E. J. Corey, J. Am. Chem. Soc. 2007, 129, 12686-12687; b) E. J. Corey, Angew. Chem. Int. Ed. 2009, 48, 2100-2117; c) B. K. Senapati, G.-S. Hwang, S. Lee, D. H. Ryu, Angew. Chem. Int. Ed. 2009, 48, 4398-4401.

[13] a) J. Yang, A. J. M. Farley, D. J. Dixon, Chem. Sci. 2017, 8, 606-610; b) A. J. M. Farley, C. Sandford, D. J. Dixon, J. Am. Chem. Soc. 2015, 137, 15992-15995; c) Y. Kobayashi, Y. Taniguchi, N. Hayama, T. Inokuma, Y. Takemoto, Angew. Chem. Int. Ed. 2013, 52, 11114-11118; d) J. Y. Kang, R. G. Carter, Org. Lett. 2012, 14, 31783181.

[14] a) S. Mayer, B. List, Angew. Chem. Int. Ed. 2006, 45, 4193-4195; b) M. Mahlau, B. List, Angew. Chem. Int. Ed. 2013, 52, 518-533.

[15] a) T. James, M. van Gemmeren, B. List, Chem. Rev. 2015 115 , 9388-9409; b) Z. Zhang, H. Y. Bae, J. Guin, C. Rabalakos, M. van Gemmeren, M. Leutzsch, M. Klussmann, B. List, Nat. Commun. 2016, 7, 12478.

[16] T. Gatzenmeier, M. van Gemmeren, Y. Xie, D. Höfler, M. Leutzsch, B. List, Science 2016, 351, 949-952.

[17] T. J. Seguin, S. E. Wheeler, Angew. Chem. Int. Ed. 2016, 55, 15889-15893.

[18] a) N. Minowa, T. Mukaiyama, Chem. Lett. 1987, 16, 17191722; b) J. Otera, Y. Fujita, S. Fukuzumi, Tetrahedron 1996, 52, 9409-9418; c) H. Yanai, O. Kobayashi, K. Takada, T. Isono, T. Satoh, T. Matsumoto, Chem. Commun. 2016, 52, 3280-3283.

[19] a) P. S. J. Kaib, L. Schreyer, S. Lee, R. Properzi, B. List, Angew. Chem. Int. Ed. 2016, 55, 13200-13203; b) Y. Xie, G.-J. Cheng, S. Lee, P. S. J. Kaib, W. Thiel, B. List, J. Am. Chem. Soc. 2016, 138, 14538-14541; c) S. Lee, P. S. J. Kaib, B. List, J. Am. Chem. Soc. 2017, 139, 2156-2159.

[20] A. G. Wenzel, E. N. Jacobsen, J. Am. Chem. Soc. 2002 124, 12964-12965.

[21] a) D. A. Evans, M. C. Willis, J. N. Johnston, Org. Lett. 1999, 1, 865-868; b) J. S. Johnson, D. A. Evans, Acc. Chem. Res. 2000, 33, 325-335.

[22] a) S. Ahmad, Tetrahedron Lett. 1991, 32, 6997-7000; b) K. Narasaka, Y. Hayashi, H. Shimadzu, S. Niihata, J. Am. Chem. Soc. 1992, 114, 8869-8885.

[23] a) M. B. Boxer, H. Yamamoto, Org. Lett. 2005, 7, 31273129; b) K. Takasu, N. Hosokawa, K. Inanaga, M. Ihara, Tetrahedron Lett. 2006, 47, 6053-6056; c) Y. Zhang, F. Lay, P. García-García, B. List, E. Y. X. Chen, Chem. Eur. J. 2010, 16, 10462-10473. 